\title{
A propos de la condition de Thoma dans les cheminées cylindriques à étranglement optimum
}

\section{On the Thoma criterion in surge tanks with optimum throttling}

\author{
PAR M. BOUYARD

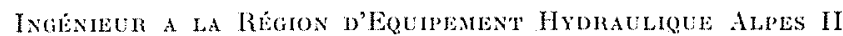 \\ D'Elactructré DE FinaNCE
}

English synopsis, p. 115.

Notre étude comportera deux parties bien distinctes :

- Dans la première, nous rappellerons un procédé de calcul des cheminées d'équilibre que M. Molbert et moi-même avons récemment proposé. Il a fait l'objet d'un article publié dans le $n^{\circ} 3$ de la Houille Blanche de 1951 , aussi nous nous bornerons à le rappeler brièvement (procédé direct). Il nous servira, dans le cadre de cette étude, à établir une formule sem! empirique donnant les sections d'une chcminée à étranglement optimum en fonction des caractéristiques de la galeric d'amenée et du niveau maximum attein! lors d'une fermeture complete instantanée.

- Dans la seconde, nous comparerons la section ainsi obtenue à celle qui serait nécessaire à l'entretien des oscillations de réglage (condition de Tromi).

\section{Rappel. du procédé direct}

Dans les calculs habituels de cheminée d'équilibre à la fermeture, on se donne les caractéristiques d'un ouvrage :

- Sections (éventuellement variables),

- Hautewr,

- Etranglement éventuel, el on examine si les limites qu'on a fixées à l'oscillation ne sont pas dépassées lors des mancuvres examinées.

Dans le procédé de calcul que nous avons appelé direct, au contraire, on prend comme inconnue la section de la cheminée, après avoir fixé les caractéristiques du mouvement :

- Montée maximum el mème valeurs intermédiaires définies par les courbes $Z_{(t)}$ et $Z_{(Q)}$;

- Débit $Q_{3}$ absorbé par les turbines.

L'application des équations habituelles permet alor's de trouver la section, analytiquement dans certains cas particuliers, graphiquement pour des cas plus complexes.

Nous en examinerons l'application au cas suivant :

- Fermeture complète instantanée;

-- Etranglement optimum (au sens donné par MM. Calame et Gaden à cette expression);

- La pression reste constante au bas de la cheminće.

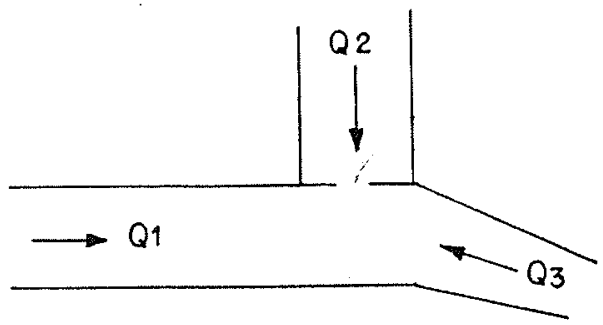


Les équations seront les suivantes :

1. Eguation de la dynamoue :

$$
\frac{\mathrm{L}}{g f} \frac{d \mathrm{Q}_{1}}{d t}=\mathrm{Z}+\mathrm{K}_{1}\left(\mathrm{Q}_{1}\right)^{2}+\mathrm{K}_{2}\left(\mathrm{Q}_{2}\right)^{2}
$$

c'est-à-dire, puisqu'il s'agit d'une fermeture supposée complète et instantanée :

$$
\frac{\mathrm{L}}{g f} \frac{d Q}{d t}=\mathrm{Z}+\mathrm{K}_{1}(\mathrm{Q})^{2}+\mathrm{K}_{2}(\mathrm{Q})^{2}
$$

L représente la longueur de la galerie,

$f$ sa section,

$Z$ le niveau dans la cheminéc, rapporté au niveau statique supposé fixe,

$K_{1}$ la constante des pertes de charge dans la galerie,

$K_{.2}$ la constante des pertes de charge à travers l'étranglement.

2" Eovation de contrnuté:

$$
\mathrm{Q} d t=-\mathrm{S} d \mathrm{Z}
$$

S représentant la section horizontale de la cheminée.

3" EQvation de la monté: :

On admettra que la montée est caractérisée par une pression constante au bas de la cheminée, ce qui s'écrit :

$$
Z+\mathrm{K}_{2} \mathrm{Q}^{2}=\mathrm{C}^{\mathrm{te}}=\mathrm{Z}_{m}
$$

$Z_{m}$ représentant la montée maximum admise dans la cheminée.
Ce système d'équation se résoud très facilement et donne notamment :

$$
\mathrm{Q}=\frac{Q_{0} K_{1} Z_{m}-Z_{m} \operatorname{tg} \frac{t}{\tau}}{K_{1} Z_{m}+K_{1} Q_{0} \lg \frac{t}{\tau}}
$$

avec :

$$
\frac{1}{\tau}=\frac{g f}{\mathrm{~L}}=\sqrt{\mathrm{K}_{1} Z_{m}}
$$

et pour la section variable en fonction de l'altitude :

$$
\mathrm{S}=\frac{\mathrm{W}^{2}}{2 g} \quad \frac{\mathrm{L} f}{\mathrm{R}^{\prime} \mathrm{Z}_{m}+\mathrm{R}_{1}\left(\mathrm{Z}_{m}-\cdots \mathrm{Z}\right)}
$$

IV représente la vitesse moyenne dans la galerie,

$R_{1}$ et $R_{2}$ respectivement la perte de charge dans la galerie et à travers l'étranglement.

Il est évident qu'on ne pourrait construire une cheminée dont la section varierait de façon continue, ni même de façon discontinue, mais fréquente. Elle présenterail, dans ce cas, l'avantage d'avoir le volume strictement minimum (pour une surpression donnéc) puisque la pression dans la galerie serait rigoureusement constante et égale à sa valeur maximum. Nous appellerons un tel ouvrage « optimorum » pour en faciliter la désignation.

Pour nous, l'intérêt de cette formule résidera surtout dans le fait qu'elle nous permettra des calculs intéressants dans le cas d'une cheminée cylindrique.

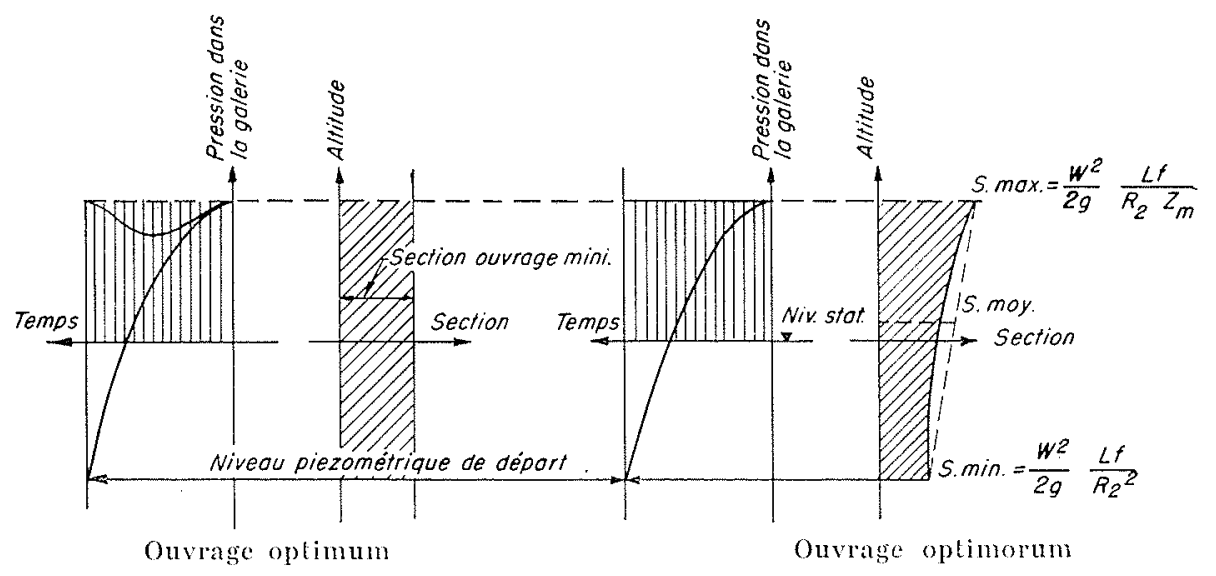

La section minimum se trouve placée au point le plus bas correspondant à $Z_{1}=-R_{1}$. Flle a comme expression :

$$
\mathrm{S}_{\min .}=\frac{\mathrm{W}^{2}}{2 g} \quad \frac{\mathrm{L} /}{\mathrm{R}_{2}{ }^{2}}
$$

et la section maximum, au point le plus haut :

$$
\mathrm{S}_{\max ,}=\frac{\mathrm{W}^{2}}{2 \mathrm{~g}} \frac{\mathrm{L} f}{\mathrm{R}_{2 .} Z_{m}}
$$

La section varie de facon hyperbolique entre $S_{\text {min. }}$ et $S_{\operatorname{mix}}$, de sorte que la moyenne arithmétique entre les deux extrêmes est supérieure à la valeur de l'ordonnce moyenne de $S_{(z)}$. Si on considère maintenant la surface de la cheminée cylindrique, simplement oṕtimum et de même montée, elle sera supérieure à l'ordonnée moyennne de l'optimorum, du fait de son efficacité moindre; on peut espérer qu'il n'y aura 
pas beaucoup d'écart entre la moyenne arithmétique de $S_{\text {min. }}$ et $S_{\max }$ et la section de la cheminée simplement optimum.

Nous allons voir que la comparaison avec les résultats classiques de Calane et Gaden montre qu'en réalité la section moyenne et la section optimum (de mème montée) sont pratiquement égales. Ce résultat est uniquement empirique.

La section moyenne a pour expression :

$$
\mathrm{S}=\frac{W^{\prime}}{2 g} \frac{\mathrm{L} f}{2 \mathrm{R}_{2}}\left[\frac{1}{\mathrm{R}_{2} \mathrm{~g}}+\frac{1}{\mathrm{Z}_{m}}\right]
$$

Pour comparer aux résultats classiques, nous allons introduire le $Z_{*}$ qui s'exprime par, dans le cadre de nos notations :

$$
\frac{Z^{2} *}{2}=\frac{\mathrm{W}^{2}}{2 g} \mathrm{~L} f \cdot \frac{1}{\mathrm{~S}}
$$

La transposition dans notre expression aboutit finalement à la relation suivante, liant $r$ et $z_{\text {max }}$

$$
\begin{aligned}
z_{\text {max }}=\frac{r}{4 r^{2}-1} \text { avec } \quad z & =\frac{Z_{\text {max }} .}{Z_{*}} \\
r & =\frac{\mathrm{R}_{2}}{Z_{*}}
\end{aligned}
$$

Nous avons comparé les résultats que donne cette formule avec ceux que l'on peut tirer des

\begin{tabular}{|c|c|c|c|c|c|c|c|c|c|c|}
\hline$r$ & 0,71 & 0,73 & $|0,76|$ & 0,78 & 0,81 & 0,85 & 0,90 & 0,96 & 1,04 & 1,16 \\
\hline$z$ (formule) & {$[0,70$} & 10,65 & $|0,59|$ & 10,55 & $0,50 \mid$ & $(0,45)$ & $\mid 0,40$ & $|0,35|$ & 10,30 & 0,25 \\
\hline z (abagues) & 0,70 & 0,65 & $0,58 \mid$ & 0,54 & $0.50 \mid$ & $0,45\}$ & 0,40 & 0,35 & 0,31 & 0,26 \\
\hline
\end{tabular}
abaques établis par les auteurs déjà cités. Les résultats sont indiqués dans le tableau ci-dessous :

La coïncidence est donc parfaite, sauf peut être pour les fortes valeurs de la perte de charge dans la galerie d'amenée qui correspondent à des cas relativement exceptionnels. Cela n'a d'ailleurs rien d'étonnant, car si cette perte de charge tend. vers zéro, la cheminée optimum tend à devenir cylindrique, et par suite, la section moyenne égale aux sections extrêmes.

La formule (1) représente, à très pen près, la section d'une cheminée cylindrique à étranglement optimum assurant une montée maximum $Z_{m}$ lors d'une fermeture complete instantanée.

\section{Application}

Munis de cette formule, nous pourrons comparer la section ainsi définie à celle qui serait nécessaire pour satisfaire le critère de Thом. Soit $Z_{m}$ la montée maximum que l'on s'est fixée. Nous caractériserons cette montée et la perte de charge dans la galerie par des coefficients sans dimension $\lambda$ el $i$. , tels que :

$$
Z_{m}=2 . \mathrm{H} \quad \mathrm{R}_{1}=\mu . \mathrm{H}
$$

$\left(R_{1}=\right.$ perte de charge dans la galerie. $)$

H représentant la chute alimentée par la salerie d'amenée. On en tire immédiatement, puisque l'étranglement est supposé optimum :

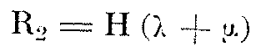

Pour que la section de THoma :

$$
\mathrm{S}=\frac{\mathrm{W}^{*}}{2 g} \frac{\mathrm{L} f}{\mathrm{HR}_{\mathrm{t}}}
$$

soit inférieure à la section nécessaire pour assurer la fermeture complète instantanée, il faudrait que l'inégalité suivante soit satisfaite :

$$
\frac{\mathrm{W}^{2}}{2 g} \frac{\mathrm{Lf}}{\mathrm{HR}_{1}}<\frac{\mathrm{W}_{2}}{2 g} \text { Lf } \frac{1}{2 \mathrm{R}_{2}{ }^{2}}\left[\frac{\mathrm{R}_{2}+\mathrm{Z}_{m}}{\mathrm{Z}_{m}}\right]
$$

soit, en faisant intervenir $\lambda$ et $s:$

$$
u^{2}(2 \lambda-1)+2 \cdot(2 \lambda-\lambda)+2 \lambda^{2}<0
$$

L'équation obtenue en égalant à zéro le premier membre de l'inégalité définit une courbe qui sépare le plan rapporté aux coordonnées rectangulaires $\lambda$, $\mu$ en différentes parties.

L'équation peut être résolue en $\lambda$ et s'écrit alors :

$$
y=-\lambda \pm \frac{\lambda}{\sqrt{1-2 \lambda}}
$$

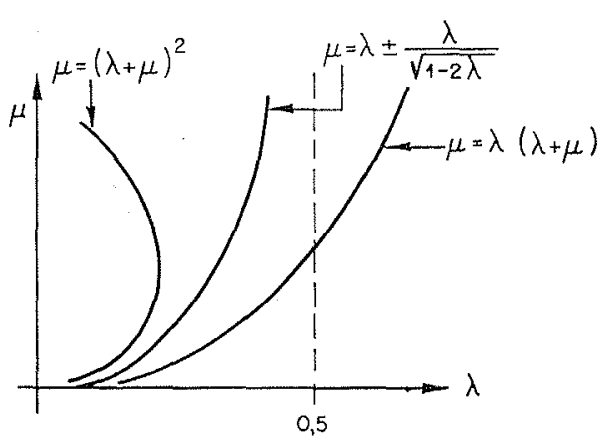

Le tracé s'en déduit immédiatement.

Nous n'avons tracé que les valeurs correspondant à $\lambda, \mu>0$ qui, seules, correspondent à des cas réels; cette courbe a l'allure ci-dessous : elle a une asymptote verticale pour $\lambda=1 / 2$ et elle est, à l'origine, tangente à l'axe des $\lambda$. 
Nous avons également indiqué les courbes limites relatives aux sections supérieure ot inférieure de la cheminée optimorum. Leurs équations sont les suivantes :

- Section minimum : $\mu=(\lambda+\mu)^{2}$ parabole à $45^{\circ}$ sur les axes,

- Section maximum :

$$
\mu=\lambda(\lambda+\mu) \text { hyperbole. }
$$

Nous donnons, ci-dessous, quelques-uns des points de la courbe principale.

\begin{tabular}{|l|l|l|l|l|l|l|}
\hline$\lambda$ & 0 & 0,10 & 0,15 & 0,20 & 0,25 & 0,30 \\
\hline$\mu$ & 0 & 0,012 & 0,0295 & 0,058 & 0,104 & 0,175 \\
\hline
\end{tabular}

Un système galerie-cheminée est caractérisé par ses deux coordonnées : :- qu'on connait une fois la galerie déterminée et $\lambda$. qu'on doit se fixer pour calculer la cheminée. Etant donné la configuration de la courbe limite au voisinage de l'origine, le point sera le plus souvent à droite de la courbe, de sorte que le critère de Thoma sera satisfait sans qu'on ait à en tenir compte dans le dimensionnement de la cheminée.

Ainsi, pour les chutes hautes et moyennes, $\lambda$ ne sera que rarement supérieur à 0,10 correspondant, pour une chute de $200 \mathrm{~m}$, à une montée, donc à une surcharge de $20 \mathrm{~m}$ par rapport au niveau statique. Or, il faut remarquer que la surpression maximum atteint, dès le début, $Z_{m}+R_{1}$; elle se manifeste en quelques secondes et peut même provoquer, dans certains cas, des coups de bélier non négligeables.

D'autre part, les canalisations en charge sont souvent constituées par une galerie en rocher ou une conduite en béton armé où on limitera la charge, par rapport au niveau statique, à une valeur assez faible (une trentaine de mètres par exemple) qu'on s'efforcera de ne pas augmenter de façon importante, lors du fonctionnement de la cheminée d'équilibre. Dans le calcul des organes d'adduction, il faut, en effet, tenir compte, non seulement de la valeur absolue des pressions auxquelles elles sont soumises, mais aussi de la rapidité de leur application. Ainsi, la valeur de correspondant à $\lambda=0,10$, que nous venons de considérer, est de 0,012 , ce qui équivaut à une perte de charge de $1,2 \%$, toujours dépassée en pratique.

Pour les chutes plus basses, ce seront, en général, les conditions de surpression dans la conduite et les turbines, ainsi que les conditions de régulation qui limiteront à moins de $20 \%$ la montée proprement dite dans la cheminée d'équilibre. Il s'ajoute encore à la surpression due à l'oscillation en masse, dès la fin de fermeture du régulateur, soit $\mathrm{H}(\lambda+\varphi)$, le coup de bélier dans la conduite d'amenée qui atteint souvent $20 \%$. Enfin, les sections des ouvrages deviennent plus importantes, ce qui accroît l'effet néfaste des surpressions. Le coefficient $y$ correspondant à $\lambda=0,20$ est égal à 0,05 , ce qui est, en général, inférieur aux pertes de charges consenties dans une canalisation d'amerée au moins pour de faibles chutes.

On peut done dire que, dans la plupart des cas, la condition de Troma sera satisfaite si la cheminée d'équilibre assure, comme c'est le cas général, la fermeture complete. En réalité, d'ailleurs, la section devra encore souvent etre renforce pour permettre les mancuves de fermeture non instantanées ou partielles, qui peuvent conduire à des montées plus importantes que les manceuvres totales.

\section{Cheminées à étranglement non optimum}

Si la cheminée est cylindrique, sa section moyenne sera nécessairement plus importante que celle de la cheminće à étranglement optimum, de même montée, et tout ce que nous avons dit s'applique à plus forte raison dans ce cas.

Si la cheminée est à section variable, notrc relation ne s'applique plus, car la section de la cheminée peut ètre quelconque et aussi petite qu'on le veut, entre le niveau piézométrique de départ et un point situé à une distance de la montée maximum égale on inférieure à la perte de charge à traver's l'étranglement, à l'instant considéré. On remarquera, cependant, que cette hauteur est, en général, relativement faible. La prise en compte de la condition de Troma ne pourrait d'ailleurs qu'exercer un effet favorable sur la surpression en la rendant plus progressive, spécialement si la prise peut être exploitée à plusieurs niveaux statiques.

Cette section ne sera pas perdue pour l'oseillation; le potentiel d'un élément $d V$, qu'on ajoutera au voisinage du niveau piézométrique, sera simplement réduit de :

$$
d V .\left(Z_{\text {max }},+R_{1}\right) \text { à } d V\left(R_{2}\right) \text {; }
$$

$d V\left(Z_{\text {max. }}\right)$ représente le potentiel d'un élément placé à la partie supérieure de l'ouvrage;

dV $\left(\mathrm{R}_{2}-\mathrm{R}_{1}\right)$ le potentiel d'un élćment ramené au voisinage du niveau piézométrique pour augmenter la section de l'ouvrage.

De toutes façons, et ce sera là notre conclusion, la prise en compte du critère de Troma serait loin de coûter aussi cher que l'augmentation apparente de section ne le laisse supposer, étant donné, par ailleurs, 'ses effets favorables sur les surpressions. Ce sera d'autant plus vrai que l'étranglement créera une perte de charge plus importante. 